\title{
Expertos e ignorantes: malas razones para el elitismo penal
}

\author{
Experts and Ignoramuses: Bad Reasons for Penal \\ Elitism
}

Especialistas e ignorantes: más razões para 0 elitismo penal

Des experts et des ignorants: mauvaises raisons pour l'élitisme criminel

专家和昧:犯罪精英的不良理由

\section{Emiliano Vitaliani ${ }^{1}$ \\ Universidad de Buenos Aires - Argentina}

Revista Derechos en Acción ISSN 2525-1678/ e-ISSN 2525-1686

Año 5/Nº 14, Verano 2019-2020 (21 diciembre a 20 marzo), 302-326

DOI: https://doi.org/10.24215/25251678e362

ORCID: https://orcid.org/0000-0003-1581-5459

Recibido: 17/12/2019

Aprobado: 01/03/2020

Resumen: nuestra Constitución consagra en su art. 39 el derecho a presentar iniciativas ciudadanas de ley. Sin embargo, estas iniciativas no pueden versar sobre algunas materias vedadas entre las que se encuentra el derecho penal. Esta restricción es tributaria de una concepción elitista del derecho penal según la cual él es especialmente complejo y las mayorías son especialmente irracionales cuando lo tratan, por lo que debería estar alejado de las pasiones populares. Este trabajo se propone mostrar que el argumento de la complejidad no es suficiente para afirmar que el derecho penal deba estar distanciado de la participación

\footnotetext{
1 Estudiante avanzado de Abogacía y Ciencia Política (UBA). Experiencia laboral en estudios jurídicos. Ayudante alumno de Elementos de derecho constitucional. Autor de "Las Comunas de la Ciudad de Buenos Aires", en Azrak, Damián (comp), Pensar la Ciudad. Comentarios a la Constitución porteña desde la igualdad, autonomía personal y derechos sociales.
} 
ciudadana. Para ello, se argumentará en primer lugar que no hay preguntas especialmente complejas en el derecho penal, sino que se trata en esencia de reprochar algunas conductas a nuestros conciudadanos de alguna forma específica. En tanto reprochamos conductas que nos parecen socialmente condenables, parecería ser que la sociedad tiene algo especialmente relevante para decir en el asunto.

Palabras clave: populismo penal- elitismo penal - democracia

Abstract: our Constitution enshrines the right to citizens' initiative. However, there are certain restrictions to this right. Some topics, including criminal law, are not allowed to be object of an initiative. This restriction derives from penal elitism, a theory that claims that penal law is too complex to be treated by ordinary citizens, who have a tendency to be irrational when it comes to discuss criminal policy. So, citizens should be far from decision making on criminal law. This paper aims to show that these arguments are not enough to support the elitist position. First, criminal law is not as complex as elitists think. Actually, its main questions are quite simple ones, since penal law is essentially about blaming our fellow citizens for some misbehavior. As we condemn those acts that we think are socially reproachable, it seems that citizens should have a special say on the matter.

Key words: penal populism - penal elitism - democracy

Resumo: Nossa Constituição consagra em seu art. 39 o direito de apresentar iniciativas cidadãos de lei. No entanto, essas iniciativas não podem ser sobre alguns assuntos proibidos entre os quais encontra-se o direito penal. Essa restrição é tributária de uma concepção elitista do direito penal de acordo com a qual ele é especialmente complexo e as maiorias são especialmente irracional quando o trata, por isso deve estar longe das paixões populares. Este trabalho visa mostrar que 0 argumento da complexidade não basta afirmar que o direito penal deve estar distanciado da participação do cidadão. Para fazer isso, vai argumentar em primeiro lugar que não há questões particularmente complexas no direito penal, senão que se trata essencialmente de reprovar alguns comportamentos de nossos concidadãos de alguma maneira específica. Embora reprochamos comportamentos que nos parecem socialmente condenáveis, parece que a sociedade tem algo especialmente relevante para dizer sobre 0 assunto. 
Palavras-chave: populismo penal - elitismo penal - democracia.

Résumé: Notre Constitution consacre dans son article 39 le droit de présenter des initiatives citoyennes des lois. Cependant, ces initiatives ne peuvent pas verser sur certaines questions interdites, parmi lesquelles il y a le droit pénal. Cette restriction est la conséquence d'une conception élitiste du droit pénal selon laquelle il est particulièrement complexe, et las majorités sont irrationnelles pour le traiter; pour cela il devrait être loin des passions populaires. Ce travail vise à montrer que l'argument de la complexité ne suffit pas à affirmer que le droit pénal doive être éloigné de la participation citoyenne. Pour cela, on soutiendra en premier lieu qu'il n'y a pas de questions particulièrement complexes dans le droit pénal, au contraire, il s'agit essentiellement de reprocher certains comportements d'une manière spécifique. Alors que nous reprochons des comportements qui nous semblent socialement condamnables, il est à prévoir que la société ait quelque chose pertinent à dire en la matière.

Mots-clés: populisme criminel - élitisme criminel - démocratie

摘要: 我们的宪法体现了其艺术精神。39提出公民法律倡议的权利。 但是, 这些举措无法处理某些违禁事项, 其中包括刑法。该限制是精 英主义刑法概念的一个附属概念，根据该概念它特别复杂，而大多 数人在对待它时尤其不理性，因此应该远离大众的热情。仅仅肯定 刑法必须与公民参与相距甚远是不够的。为此, 首先要论证的是, 刑 法中没有特别复杂的问题，但本质上是关于以某种特定方式向我们 的同胞提出某些行为。当我们谴责那些在社会上应受我们谴责的行 为时, 社会似乎对此事特别有话要说。

关键词: 犯罪民粹主义, 犯罪精英主义, 民主

\section{Introducción}

Un espectro se cierne sobre el derecho penal: el espectro del populismo penal. Contra este espectro se han conjurado muchos de los mejores teóricos de nuestra disciplina y también nuestra Constitución. El populismo penal hizo su aparición a fines del siglo pasado y desde entonces este fenómeno no perdió centralidad. Si bien delimitaré sus contornos con mayor 
precisión más adelante, podemos muy sucintamente decir que se caracteriza por contener un conjunto de demandas punitivistas y por originarse en reclamos que la ciudadanía realiza a sus representantes. Estos reclamos pueden canalizarse tanto a través del sistema representativo como mediante iniciativas ciudadanas de referéndum. Ante esta ola punitivista, distintos autores han reaccionado promoviendo la restricción de la participación ciudadana en cuestiones penales.

Nuestra Constitución Nacional parece reflejar este debate. En la reforma de 1994 se decidió incorporar a nuestro texto constitucional algunos mecanismos de democracia semidirecta. Así, el art. 40 de la Constitución reformada permite la realización de consultas populares vinculantes y no vinculantes, y el 39 incorpora las iniciativas ciudadanas de ley. Sin embargo, si bien la regla que permite las iniciativas ciudadanas es de carácter general, ella cuenta con algunas excepciones entre las que se encuentra la materia penal. Es decir que incluso si un conjunto de ciudadanos cumpliera los requisitos que manda el ordenamiento jurídico para presentar un proyecto de ley, ello no sería posible si esta ley tuviera por objeto el derecho penal.

Esta restricción a la participación ciudadana en materia penal fue defendida tanto en la Convención Constituyente como en la literatura especializada en virtud de la complejidad que reviste el derecho penal y las pasiones que desata. El objetivo de este trabajo es mostrar que la restricción en materia penal que surge del art. 39 no podría ser sostenida sobre la base de estos argumentos incluso si se aceptaran las premisas teóricas que lo sustentan. Por supuesto, una misma regulación puede ser defendida en virtud de más de un argumento, por lo que rechazar el argumento de la complejidad no implica el rechazo pleno a la restricción del art. 39. Simplemente me propongo aquí ofrecer razones para descartar este argumento como fundamento de la prohibición a las iniciativas ciudadanas de ley en materia penal.

El trabajo estará organizado en dos partes centrales además de esta introducción y la conclusión. En la primera reconstruiré 
el argumento de la complejidad, ya que es a partir de su acabada comprensión que podremos más adelante detectar sus falencias. Allí veremos que el elitismo penal se desarrolla por oposición al populismo penal, un fenómeno extendido de participación popular que rechaza a las elites políticas e intelectuales a la vez que reclama una política criminal más dura.

La segunda parte del trabajo tendrá como objetivo desacreditar los argumentos reconstruidos anteriormente. Se afirmará allí que incluso si supusiéramos que existen algunas cuestiones que por su complejidad deben ser tratadas por expertos, el derecho penal no es una de ellas. Asimismo, si estuviéramos equivocados sobre el grado de complejidad del derecho penal y su regulación fuera una cuestión de una complejidad tal que los expertos deberían ocupar un rol central en el proceso de toma de decisiones, eso seguiría estando garantizado si se permitieran las iniciativas ciudadanas en materia penal.

No pretendo discutir en las páginas que siguen el argumento según el cual algunos temas deben estar reservados a los expertos, su abordaje excedería en mucho el marco de este trabajo. Simplemente quiero aquí demostrar que incluso si aceptáramos este argumento en abstracto, al momento de aplicarlo al derecho penal este seguiría formando parte del grupo de temas que deben estar abiertos a la participación democrática.

\section{Mejor no participar en ciertas cosas}

En este apartado intentaré brindar algunas precisiones sobre este fenómeno preocupante y novedoso al que llamamos populismo penal para luego reconstruir los argumentos elitistas que sustentan restricciones como las del art. 39. Dado que el elitismo penal se construye en espejo al populismo, comenzaré por este último.

Una de las primeras características del populismo penal es su emocionalidad y su irracionalidad (Pratt, 2007: 12-15; Pratt y Miao, 2017; Sparks, 2000). Este fenómeno suele surgir a partir de hechos puntuales que desatan el enojo de una parte relevante de la ciudadanía que, por lo tanto, reclama en los medios y 
en las calles que se cambie la política criminal. Los cambios solicitados rara vez están sostenidos sobre estudios empíricos o el conocimiento de los especialistas. Por el contrario, los movimientos populistas en materia penal se caracterizan por tener reclamos basados en el sentido común y el enojo (Pratt y Miao, 2017: 44-45), rechazando el conocimiento experto de lo que consideran elites.

Esta separación entre elites y ciudadanía es otra de las características centrales del populismo penal (Dzur, 2010: 356-357; Pratt, 2007: 5-18). Al sentir que las elites no los protegen de los delincuentes, los ciudadanos reaccionan a través de movimientos populistas que ponen su foco en formas más participativas de democracia. Resultan ejemplificadores en este sentido los casos de California y Nueva Zelanda ${ }^{2}$, en donde los movimientos sociales que reclamaban una política criminal más punitivista lograron canalizar sus demandas a través de iniciativas ciudadanas de referéndum.

La última característica que atribuiremos aquí al populismo penal es la centralidad de los derechos de las víctimas. Tal como afirma Pratt, "el populismo penal nos exige que los derechos de las víctimas y la comunidad tengan preeminencia sobre los derechos de los criminales individuales" (2007: 30). Las demandas aquí enmarcadas suelen tener su origen en reclamos de familiares que son recogidos por la ciudadanía. Por lo tanto, son sus derechos los que estos movimientos buscan proteger y garantizar, lo que trae aparejado que sus demandas suelan ser de corte punitivista.

Ante este panorama escalofriante de masas irracionales reclamando más poder punitivo se levantó lo que llamamos elitismo

2 En 1994 California sancionó mediante un referéndum convocado a través de una iniciativa ciudadana una ley según la cual aquellas personas que delinquieran por tercera vez debían pasar al menos veinticinco años en prisión. En el año 1999 Nueva Zelanda también endureció su política penal como consecuencia de una iniciativa ciudadana de referéndum que concluyó con la sanción de una reforma del sistema criminal que ponía un mayor énfasis en los derechos de las víctimas e imponía trabajo forzoso a los condenados. Esta tendencia se mantuvo, incluyendo reformas sobre el régimen de libertad condicional, el contenido de las garantías penales durante la investigación y una ley del tipo three strikes como la de California (Heibert y Kelly 2015: 185-232) 
penal. El elitismo penal afirma que la formulación de las leyes penales debería estar alejada de las manos de la ciudadanía y reservada a un grupo selecto de ciudadanos que cuente con algún tipo de conocimiento especial.

La ya mencionada restricción del art. 39 parecería estar cubierta por este paraguas teórico. Si bien él acepta las iniciativas ciudadanas de ley en general, considera que hay algo especial en el derecho penal que amerita hacer una excepción. Qué es eso que hay de especial en el derecho penal es lo que intentaré dilucidar en lo que resta de este apartado. Para ello indagaré en los argumentos que justifican esta restricción. En primer lugar, tomaré los argumentos brindados en la convención constituyente que redactó el art. 39, aunque dada la precariedad de las razones allí expuestas complementaré su reconstrucción con formulaciones más sólidas realizadas por estudiosos del tema.

\section{Un asunto muy complejo}

La prohibición que rige las iniciativas ciudadanas de ley en materia penal casi no fue justificada en la Convención Constituyente en la que surgió. Por el contrario, solamente hay una breve mención del miembro informante del dictamen de mayoría, el convencional Brusca, quien afirma que el derecho penal tiene "una complejidad tal que resulta difícil que se vote por sí o por no dentro de un proyecto aislado". A los fines de poder discutir esta restricción, tomaré el argumento de la complejidad y lo desarrollaré utilizando la literatura disponible.

El argumento de la complejidad es un argumento muy frecuente en estos días. En el ámbito doméstico, Raúl Zaffaroni defendió recientemente la idea según la cual el derecho penal debe estar reservado a los expertos. En este sentido afirmó que "No se puede pretender que el Código Penal salga de la voz del pueblo"3.

\footnotetext{
3 Ver "Zaffaroni pidió no debatir el nuevo Código Penal 'en la esquina o en los medios de comunicación'". Disponible en https://www.infobae.com/2014/04/21/1558841-zaffaronipidio-no-debatir-el-nuevo-codigo-penal-en-la-esquina-o-los-medios-comunicacion/. Última vez consultado el 01/11/2019
} 
Asimismo, y mostrando su adhesión al argumento de la complejidad, también sostuvo que aceptaría críticas al anteproyecto de Código Penal del que fue corredactor siempre que ellas se hicieran en un marco de "seriedad y tecnicismo" (Zaffaroni, 2014). De esta forma, parecería que los únicos interlocutores válidos son aquellos que son técnicos en la materia y que por lo tanto pueden comprender una cuestión tan compleja como el derecho penal.

Trataré aquí de presentar las versiones más refinadas de argumentos como los brindados por Zaffaroni y el convencional Brusca. El razonamiento según el cual el derecho penal no debe quedar en manos de la ciudadanía asume que para que las decisiones políticas sean legítimas, ellas deben contar con un cierto grado de confiabilidad epistémica (Strandbrinck, 2018: 7). Sabemos que el testimonio de las personas con una expertise particular es una buena razón para dotar a una afirmación de valor epistémico (Goldman y O'Connor, 2019). Por ejemplo, en nuestra vida cotidiana actuamos dotando de valor a lo que los médicos nos dicen. Si nuestro médico de cabecera nos receta un medicamento para una enfermedad y un desconocido sin conocimientos de medicina nos dice que tomarlo sería dañino para nuestra salud, simplemente ignoramos las palabras del extraño y confiamos en las del médico. Llamamos a las personas que conocen más que un ciudadano medio sobre una determinada cuestión expertos. Dado que existen expertos en derecho penal, parecería razonable otorgarles un lugar especial o delegar en ellos su redacción si nos interesa que la misma sea correcta (Schudson, 2006: 494).

Sin embargo, este argumento no termina de explicar que existan restricciones en materia penal y no en todo el derecho. Más aún, siguiendo este razonamiento podríamos decir que no se justifica la producción democrática de leyes y que ellas deben quedar en manos de los expertos en cada uno de los temas sobre los que legislemos. Esto no es lo que se afirmó en nuestra convención constituyente (que de hecho incorporó las iniciativas ciudadanas) ni es una afirmación aceptable para una 
persona que tenga el más mínimo compromiso democrático, incluso si cree que la democracia tiene un valor epistémico. Por el contrario, parecería que hay algo especial en el derecho penal que lo hace especialmente sensible a este razonamiento. Este algo es su especial complejidad.

Si bien todas las decisiones involucran un cierto grado de complejidad en el sentido de que necesitamos algún conocimiento para poder tomarlas, el argumento de la complejidad asume que no todas nos exigen ser tomadas por un experto. Por lo tanto, vemos que para quienes sostienen que las personas con mayor expertise deben tener un lugar especial en la toma de decisiones, algunas decisiones requieren conocimientos técnicos muy específicos y en consecuencia deben ser tomadas por expertos, mientras que otras tienen un nivel bajo de complejidad y por lo tanto pueden ser tomadas por ciudadanos promedio. Según el argumento acá expuesto, el derecho penal cae bajo el primer grupo.

Cabe ahora preguntarnos por qué, cuáles son las razones que nos permiten sostener que el derecho penal es algo muy complejo y que por lo tanto debe quedar en manos de los expertos. Phillip Petit (2002) parece creer que lo que caracteriza al derecho penal es que él está signado por una dinámica de producción particular, la dinámica del escándalo (outrage dynamic). Esta dinámica es muy similar a lo que describimos más arriba como populismo penal. En ella, ante un evento particular se dispara un escándalo público que genera presión sobre los legisladores, quienes se ven obligados a responder a estas demandas. Por lo tanto, lejos de ser el producto de una calma deliberación, la legislación penal sería consecuencia de las presiones populares.

La respuesta de Petit a esta problemática es la creación de una institución similar al Banco Central en la cual confluyan expertos y representantes de organizaciones especializadas. Ellos recibirían mandatos muy generales de parte de los poderes políticos, pero redactarían la legislación con un grado relevante de autonomía. Una institución de este tipo permitiría evitar las 
presiones populares propias de la dinámica del escándalo y por lo tanto contar con una legislación confiable.

Aunque de forma menos sistemática, también Daniel Pastor detecta una tendencia neopunitivista en nuestro derecho penal contemporáneo y la atribuye al populismo penal. El populismo penal estaría caracterizado por el rechazo al conocimiento de los expertos y por su marcado contenido punitivista, contrario al derecho penal liberal que Pastor defiende (2005: 54-77). Se sigue de esta afirmación que en tanto la participación popular lleva al populismo penal y este a políticas criminales punitivistas, deberíamos rechazarla y por lo tanto restringir las formas en las que la ciudadanía puede influir sobre el derecho penal.

En un sentido similar, el jurista italiano Luigi Ferrajoli rechaza la participación ciudadana en el ámbito penal. Para ello parte de una definición de democracia que no sólo incluye al autogobierno colectivo, es decir la posibilidad de que una comunidad se dé sus propias normas, sino que su forma de entender a la democracia incluye algunos compromisos que exceden el procedimiento de toma de decisiones e incluye compromisos sustanciales. Dado que esta forma de entender a la democracia no se limita al procedimiento, sino que incluye ciertos derechos, Ferrajoli caracteriza a su definición de democracia como sustancial (2008: 77-85).

Si partimos de que la democracia incluye ciertos derechos que no pueden ser violados, aunque exista una decisión mayoritaria en contra no caracterizaremos a esta decisión como democrática y por lo tanto ella carecerá de legitimidad. Así, existen ciertas decisiones que no pueden ser tomadas. Ferrajoli llama al conjunto de estas decisiones esfera de lo indecidible (2008: 102-104).

Nos encontramos así con dos conjuntos de decisiones, uno sobre el que las mayorías no pueden influir y otro sujeto a las decisiones que surjan de los órganos representativos. Mientras que este segundo conjunto de decisiones debe quedar en manos de los poderes políticos, el primer grupo está en manos 
de los órganos aplicadores de derecho en virtud del carácter cognitivo de sus funciones (Ferrajoli 2008: 106-107). Así, parecería que Ferrajoli considera que las cuestiones de derechos son cuestiones técnicas, complejas, y que por lo tanto ellas deben quedar en manos de los poderes que estén alejados de las pasiones que rigen a la política, ya que la política democrática (entendida como gobierno de la mayoría y no en los términos de Ferrajoli) degenera inevitablemente en el gobierno de los peores (2008: 88).

Siguiendo este razonamiento, solamente queda un paso para mostrar que el derecho penal debería estar alejado de los poderes democráticos. Este paso es afirmar que el derecho penal forma parte de la dimensión sustancial de la democracia, por lo que pertenece a la esfera de lo indecidible y debe estar alejado de los poderes políticos. Ferrajoli da este paso cuando afirma que el derecho penal se trata de ofrecer garantías a los ciudadanos frente al poder del Estado, por lo que estamos en presencia de derechos inviolables que pertenecen al espacio de lo indecidible (1998: 864-866). La conclusión obvia de lo dicho hasta aquí es que el derecho penal es una cuestión de derechos y que por lo tanto debe quedar reservada a quienes tienen capacidades cognoscitivas especiales, es decir a los expertos.

Más allá de las particularidades de los desarrollos y propuestas de cada uno de los autores aquí mencionados, queda claro que existe en el derecho penal una preocupación general por el populismo penal que se traduce en propuestas de contenido elitista. Estas propuestas parten del espanto ante un fenómeno que se reproduce en todo el mundo, caracterizado por su rechazo a las elites y su pedido de endurecimiento de las penas.

Contra ese movimiento, una parte relevante de la academia jurídica afirma que en tanto el derecho penal es un asunto sumamente complejo y a su vez despierta las pasiones punitivistas de las personas, él debe quedar alejado de la participación ciudadana. Caracterizamos a estas propuestas como elitistas porque mientras que promueven el alejamiento del derecho penal de la ciudadanía, promueven que él sea dejado en manos de unos 
pocos expertos, ya sean las personas con capacidades cognitivas especiales de Ferrajoli o los técnicos de Zaffaroni.

\section{Argumentos insuficientes}

En lo que resta de este trabajo intentaré mostrar que los argumentos brindados en defensa del elitismo penal no alcanzan para sustentar dicha posición. Por el contrario, tanto argumentos teóricos como prácticos nos permiten desacreditarla. El objetivo aquí no es afirmar que no es posible defender una posición elitista, sino simplemente mostrar que incluso si aceptáramos en abstracto la premisa de que algunas cuestiones deben ser decididas por los expertos, el argumento de la complejidad seguiría siendo insuficiente para el caso del derecho penal y por lo tanto debe ser descartado.

\section{¿Es realmente tan complejo?}

Dijimos hasta acá que algunas cuestiones son tan complejas que deberían ser alejadas de la ciudadanía para quedar en manos de unos pocos expertos que pudieran deliberar con independencia de las pasiones mayoritarias. A su vez, afirmamos que lo que hace que el derecho penal caiga bajo este argumento no es tanto su complejidad específica sino la dinámica política que usualmente se genera en torno a él. Así, dado que el derecho penal desata los impulsos punitivistas de las mayorías, debemos restringir su participación en la formulación de leyes penales.

En primer lugar, cabe destacar que este razonamiento es un clásico caso de falacia naturalista. En él se parte de una afirmación descriptiva (las mayorías se guían por pasiones punitivistas) para concluir una proposición normativa (debemos limitar la participación popular en derecho penal). Creo que incluso si sus premisas fueran verdaderas, de ellas no se sigue que la participación ciudadana en la formulación de normas penales no sea deseable. Para ser válido, este razonamiento debería incorporar algunas de las siguientes proposiciones prescriptivas que paso a analizar. 
Podríamos en primer lugar afirmar que debemos adoptar la forma de toma de decisiones que nos lleve a políticas lo menos punitivistas posibles. Si eso fuera así y lográramos demostrar que las mayorías son más punitivistas que los expertos, entonces la mejor forma de toma de decisiones sería a través de los expertos.

Sin embargo, este argumento tiene un problema al considerar su relación con la democracia. La democracia, y en particular una concepción epistémica de la democracia deliberativa, supone que no conocemos cuales son las mejores políticas públicas existentes. Dado que nuestra racionalidad es limitada (Fearon, 1998: 49-52), debemos aceptar que siempre podemos estar equivocados y que por lo tanto nuestras decisiones siempre serán provisionales, no siendo aceptable un compromiso inamovible con ningún principio particular más que la misma provisionalidad (Gutman y Thompson, 2004: 110-119). Por el contrario, disponer como premisa, es decir como un elemento invariable del razonamiento, una política determinada y ajustar la forma de toma de decisiones a ella implicaría contradecir este principio, ya que nunca podríamos abandonar nuestro compromiso con una política no punitivista.

La otra premisa que podríamos adoptar para que el razonamiento no quede desacreditado por ser una falacia naturalista es ya no uno consecuencialista sino uno deontológico. En este argumento, desarrollado más arriba, asumimos que la autoridad política tiene una dimensión epistémica y que por lo tanto los expertos tienen que tener un lugar central en la toma de decisiones que requieran un alto nivel de expertise.

Sin embargo, el derecho penal no es una cuestión tan técnica como parece. Las preguntas centrales del derecho penal no son preguntas que un ciudadano común se encuentre incapacitado para responder. Si bien solo los expertos conocen los pormenores de la teoría del delito o los detalles de los tipos penales en particular, todos podemos tener opiniones acabadas 
sobre qué conductas deben ser reprochadas ${ }^{4}$. Las preguntas por qué reprochar y cómo reprochar son el núcleo del derecho penal sustantivo y ellas no requieren ningún conocimiento por demás complejo que se adquiera en la facultad o en estudios profundizados.

Si bien es cierto que existen en el derecho penal algunas cuestiones que revisten un alto grado de complejidad, no lo es menos que estas cuestiones no conforman su núcleo. Por el contrario, el derecho penal se trata en esencia de reprochar a nuestros conciudadanos conductas que nos parecen condenables (Duff, 2015a: 45-46). En términos de Duff: "el derecho penal determina que ciertos tipos de daños, preexistentes, sean considerados males públicos" (2015b: 33). En tanto todos formamos parte de la comunidad que reprocha a quien ha concretado este mal público, parece sumamente razonable pensar que todos tenemos algo valioso para decir sobre cuáles son esas conductas que estarán sujetas a reproche y qué forma debe adoptar este reproche. Por lo tanto, la formulación del derecho penal no parece ser una cuestión que por su naturaleza deba estar reservada a los expertos. Por el contrario, dado que lo que se encuentra en juego son los valores de una comunidad política, es ella y no una elite aislada quien se encuentra mejor situada para poder expresarlos y así crear un derecho penal acorde a los mismos.

Dijimos al describir los fundamentos del elitismo penal que algunas cuestiones eran especialmente complejas y que por lo tanto su abordaje debía ser dejado en manos de los expertos. Sin embargo, este no es el caso del derecho penal. No hay en sus preguntas centrales tecnicismos que sean inaccesibles para los ciudadanos ni asuntos sobre los cuales cada uno de nosotros no tenga algo valioso para decir.

\footnotetext{
4 Asistimos en estos tiempos a un debate amplísimo sobre la legalización de la interrupción voluntaria del embarazo, lo que implica la derogación del art. 86 de nuestro Código Penal, sin que haya sido necesario para que el debate fuera de calidad que todos los que participaban fueran expertos en derecho penal.
} 
Una vez descartado el argumento de la complejidad técnica del derecho penal, nos resta analizar el argumento de la irracionalidad pasional de las mayorías. En primer lugar, podríamos decir que si las masas están compuestas por personas y las personas se ven emocionalmente afectadas cuando está en juego el derecho penal, de forma tal que esto les impide razonar con claridad, esto también debería ser cierto para los expertos. Nada nos garantiza que, incluso teniendo amplios conocimientos sobre derecho penal, los expertos no se vean también afectados por la supuesta emocionalidad que conlleva el tratamiento del derecho penal.

En segundo lugar, e incluso suponiendo que el estudio materialice en los expertos una coraza que los proteja de la emocionalidad que trae aparejada el derecho penal, del hecho de que las formas actuales de participación ciudadana sean pasionales no se sigue que ello no pueda cambiar. Por el contrario, la literatura empírica muestra que la forma en la que se diseñan la participación y la deliberación colectiva tiene efectos sobre los resultados (Curato, Dryzek, Selen, Ercan, Hendriks, y Niemeyer, 2017; Fung, 2003). Por lo tanto, prohibir las iniciativas ciudadanas de ley en materia penal no es la única forma de prevenir la irracionalidad de las masas, sino que bastaría con diseñar un mecanismo deliberativo que promueva la discusión pública basada en razones.

Concluimos así que no es cierto que haya alguna particularidad en el derecho penal que lo haga inaccesible para el ciudadano promedio. Por el contrario, el derecho penal de una comunidad es un reflejo de sus valores y de la forma en la que ella resuelve sus conflictos, por lo que es deseable que los ciudadanos que la conforman se involucren en su redacción.

\section{Todos podemos conocer}

Dijimos hasta acá que el argumento elitista fallaba porque afirmaba que redactar el derecho penal requería de la participación de expertos cuando sus preguntas centrales son accesibles 
para cualquiera y requieren conocer los valores fundamentales de una determinada comunidad, tarea para la que la propia comunidad parece bien preparada. Quisiera ahora discutir otro argumento, uno relacionado no con el grado de complejidad del derecho penal sino con la capacidad de los ciudadanos de adquirir conocimiento a través de la deliberación pública.

El elitismo divide a la sociedad entre expertos y no expertos. Según esta corriente estos grupos están claramente demarcados y son identificables. Sin embargo, lo que el argumento elitista no considera es que las personas pueden adquirir conocimientos durante un proceso deliberativo, por lo que evaluar la expertise de los ciudadanos en un momento anterior a la deliberación colectiva constituye un error.

La teoría de la democracia deliberativa considera que las posiciones que las personas tienen sobre una determinada cuestión están influenciadas por el proceso de toma de decisiones. Para esta teoría las personas no tienen preferencias fijas y autointeresadas sino que en la vida pública actuamos de acuerdo a otros criterios (Elster, 1997). Por lo tanto, no entendemos aquí a la democracia como la mera agregación de preferencias individuales fijas y previamente determinadas, sino que el proceso que precede a esta agregación también tiene un rol fundamental. Al deliberar las personas pueden conocer tanto los principios de moralidad pública defendidos por sus conciudadanos y los argumentos teóricos y empíricos que los sostienen, así como defender los propios principios ante los demás. De esta forma cada uno adquiere nueva información a la vez que se ve obligado a brindar razones públicamente aceptables para defender las propias posiciones (Rawls, 1997), aumentando la confiabilidad epistémica de las decisiones.

Si lo dicho hasta aquí es cierto, las personas llegamos más informadas al momento de decidir que lo que estábamos al momento de iniciar la deliberación. A través del proceso deliberativo aumenta nuestra expertise sobre el tema en cuestión, por lo que evaluar la diferencia entre el conocimiento de los 
expertos y de los no expertos al momento en que comienza el proceso es un error.

No se afirma aquí que no existan diferencias de conocimiento relevantes entre expertos y no expertos, tampoco que incluso después de un proceso deliberativo los ciudadanos sean catedráticos de derecho penal. Sin embargo, ellos sí pueden a través de la deliberación pública conocer los asuntos más relevantes que conciernen a la legislación penal.

Thomas Christiano (2013: 37-42) identifica algunos mecanismos mediante los cuales se puede democratizar la división del trabajo entre expertos y no expertos propia de un sistema deliberativo. Dado que es el único que permite transmitir información de los expertos a los no expertos, me concentraré aquí en lo que el autor denomina entendimiento superpuesto (overlaping understanding).

El entendimiento superpuesto parte del supuesto de que muchas veces dos personas comparten algún área de expertise pero no otra. Así, si el sujeto $\mathrm{P}$ es experto en las cuestiones $a$ y $b$, mientras que el sujeto Q es experto en $a$ y $c$. Dado que $\mathrm{P}$ y $\mathrm{Q}$ comparten el área de conocimiento $a, \mathrm{P}$ puede explicarle a $\mathrm{Q}$ su conocimiento sobre $b$ en términos de $a$, ya que ambos los conocen. Una vez que adquirió este nuevo conocimiento, $\mathrm{Q}$ puede transmitirlo a todos los que conozcan $c$, lo que habría sido imposible para P. De esta forma el conocimiento experto que tal vez en un primer momento fuera inaccesible para los ciudadanos no expertos se torna aprehensible cuando este mecanismo se da de forma repetida. Tal como afirma Christiano (2013: 39): "las cadenas de inteligibilidad superpuesta permiten a políticos y ciudadanos tener algún conocimiento de las razones existentes a favor y en contra de una determinada política pública".

Por lo tanto, el elitismo falla al creer que la expresión agregada de las opiniones individuales es análoga a una decisión democrática. Si la democracia es también el proceso que precede a la toma de decisiones (y no cualquier proceso sino uno que nos permita intercambiar razones), y en este proceso los expertos 
pueden transmitir conocimiento a los no expertos, entonces ella no solo refleja, sino que moldea nuestras preferencias (Gargare1la, 2016: 218-223). Al deliberar, los ciudadanos estamos expuestos a información y argumentos que antes ignorábamos, por lo que nuestro conocimiento sobre el objeto de debate aumenta. Así, la brecha entre expertos y no expertos se reduce a través de la discusión, permitiéndonos satisfacer el estándar epistémico que tal vez no pasáramos al iniciar el proceso.

\section{Los expertos sí participan}

Por último, quisiera agregar un argumento ya no teórico sino de tipo empírico. Afirmaré que si se permitieran las iniciativas ciudadanas de ley en materia penal ${ }^{5}$, los expertos no dejarían de tener un rol fundamental tanto al nivel de la redacción como en la sanción del derecho penal.

Al nivel de la decisión sobre la adopción de una determinada legislación penal, nuestra Constitución acepta al Congreso como una institución lo suficientemente preparada para decidir. Si bien en algunas oportunidades la redacción del derecho penal es delegada a una comisión de especialistas, la adopción o no de estos proyectos o anteproyectos de ley es una decisión que queda en manos de nuestros representantes.

Esto mismo es lo que sucede con las iniciativas ciudadanas en nuestro contexto normativo actual. De acuerdo a nuestra legislación la iniciativa de ley es presentada al Congreso, quien decide si adoptarla o rechazarla, es decir que es el órgano representativo quien tiene la última palabra sobre la adopción del texto propuesto.

Asimismo, dijimos que la Constitución prevé que el Congreso pueda tanto redactar como sancionar derecho penal, por lo que podemos asumir que este cuenta con un nivel de

\footnotetext{
5 En nuestro ordenamiento jurídico solamente se permiten las iniciativas ciudadanas de agenda, es decir aquellas en las que el Congreso tiene la facultad de decidir si acepta 0 rechaza el proyecto de ley.
} 
expertise satisfactorio. La idea misma de representación política en el sentido actual tiene como objetivo que quienes nos gobiernen sean personas con cualidades especiales, lo que permitiría seleccionar a una elite que tome las mejores decisiones posibles (Manin, 1997). En este sentido, resultan esclarecedoras las palabras de Madison, quien afirma que la representación es el mecanismo mediante el cual la voluntad popular pasa por el "tamiz de un grupo escogido de ciudadanos, cuya prudencia puede discernir mejor el verdadero interés de su país" (Hamilton, Madison y Jay, 2014: 39). Así, podríamos considerar que la representación promueve que los legisladores tengan una expertise superior a la media.

Incluso suponiendo que nuestros representantes no sean expertos cuando son electos, lo que es posible dado que no hay nada en los requisitos impuestos para su elección que lo garantice, ellos podrían estar especialmente bien situados para decidir desde el punto de vista de la expertise como consecuencia del ejercicio de su cargo. Si bien los legisladores pueden acceder sus cargos sin conocimientos de derecho penal, el ejercicio reiterado de la actividad legislativa acrecienta el conocimiento sobre los asuntos públicos (Hamilton, Madison y Jay 2014: 230). No sólo los representantes cuentan con el tiempo necesario para adquirir conocimiento, sino que también son asesorados por especialistas en cada una de las materias legislativas. Vemos entonces que al nivel del Congreso los expertos actúan de dos formas distintas. Por un lado, ellos asesoran a quienes luego tomarán decisiones y por otro proveen conocimiento a los legisladores, quienes así adquieren su calidad de expertos si no la tenían al momento de su elección.

Podemos distinguir así entre dos tipos diferentes de expertos. Por un lado, algunos son expertos en virtud del conocimiento adquirido en espacios de educación formal, mientras que por otro encontramos expertos democráticos. Este segundo grupo está constituido por aquellas personas que cuentan con una expertise particular pero que a su vez están democráticamente legitimadas. 
Ahora bien, si según nuestra Constitución el Congreso está bien situado para tomar decisiones en materia de derecho penal y si todas las iniciativas de ley deben pasar por el Congreso, entonces es imposible evitar el filtro de los expertos. Dado que el Congreso debe intervenir en todos los casos, es falso que las iniciativas ciudadanas de ley en materia penal consagren el derecho a legislar de los no expertos. Para poder sancionar una ley siempre habrá que pasar por el filtro parlamentario, es decir por el filtro de lo que nuestra Constitución consideró expertos. Por lo tanto, permitir las iniciativas ciudadanas de ley en materia penal no sería quitar la decisión de sus manos, sino simplemente aceptar que el objeto de esa decisión puede tener su origen en la ciudadanía.

Como dijimos, permitir a los ciudadanos tener iniciativa de ley en materia penal no excluiría a los expertos, pero el nivel del Congreso no es el único en el que los expertos estarían incluidos en la sanción de la legislación penal. Hasta acá hablé de ciudadanos y expertos como si fueran dos conjuntos de personas que no se tocan entre sí. Sin embargo, esto es un error en muchos sentidos. En primer lugar y de forma más obvia, podemos decir que los expertos son ciudadanos, por lo que ellos tienen alguna participación en las iniciativas ciudadanas de ley. Sin embargo, y aunque esto sea cierto, si lo que queremos afirmar es que los expertos participarían en la redacción de leyes penales de permitirse las iniciativas, lo que en realidad estamos queriendo afirmar es que ellos tendrían un lugar especial. Así, no nos basta con que ellos intervengan como cualquier otra persona, sino que queremos que lo hagan en condición de expertos.

Por lo tanto, debemos ahora preguntarnos si los expertos participarían en condición de tales si se permitieran las iniciativas ciudadanas de ley en materia penal. Por supuesto, no estamos en condiciones de saber con seguridad qué pasaría si esta posibilidad se habilitara, dado que hacerlo sería metodológicamente erróneo. Sin embargo, esto no nos impide recurrir a ciertos indicios que nos permitan tener sospechas fundadas. 
Sabemos que las iniciativas de ley suelen ser promovidas por movimientos sociales más o menos articulados. Estos movimientos sociales generalmente persiguen sus objetivos a través de las vías que consideran más efectivas. En este sentido, sabemos que muchas veces estas vías son el litigio estratégico y el activismo judicial. Por lo tanto, los movimientos sociales y el derecho no tienen lógicas excluyentes, sino que se influyen mutuamente, lo que ha llevado a que los abogados se involucren de diversas formas en la defensa de sus reclamos (Sarat y Scheingold, 2006). Vemos así que los abogados, lejos de estar disociados de los movimientos sociales, tienen un rol central en ellos, en particular cuando se trata de cuestiones jurídicas.

En virtud de lo expuesto hasta acá, concluyo que lejos de excluir a los expertos de la toma de decisiones, permitir las iniciativas ciudadanas de ley en materia penal incluiría nuevos actores en la producción del derecho sin que ello implique que los expertos queden a un costado. Ellos no solo podrían participar al nivel del Congreso tanto como representantes como en calidad de asesores, sino también al nivel de la sociedad civil, ya que los abogados usualmente se involucran en movimientos sociales, los que dan a los expertos un lugar central cuando se trata de cuestiones jurídicas.

\section{Conclusión}

El objeto de estudio de este trabajo fueron los argumentos que justifican la exclusión de la materia penal de los asuntos sobre los cuales los ciudadanos pueden presentar iniciativas de ley según el art. 39 de nuestra Constitución, planteando como hipótesis que ellos eran insuficientes. Dado que para poder analizar algo uno tiene que en primer lugar saber en qué consiste ese algo, el primer objetivo fue reconstruir estos argumentos. Para hacerlo, examiné los debates de la Convención Constituyente que dio lugar a la reforma del '94, en la cual se incorporó el artículo bajo examen. 
En estos debates casi no se ofrecieron razones para restringir las iniciativas en materia penal, aunque sí existió una pequeña mención del miembro informante del dictamen de mayoría a la complejidad del derecho penal. El argumento de la complejidad parte de la asunción de que algunos temas deben estar alejados de la participación ciudadana y estar reservados a los expertos. Así, afirma que la redacción del derecho penal debe estar reservada a los técnicos, ya que ellos son los únicos capacitados para tratar con un objeto tan complejo.

En lugar de discutir los presupuestos teóricos que sustentan esta afirmación, me propuse mostrar que no se sigue de allí que el derecho penal deba estar alejado de la ciudadanía. Es decir que incluso aceptando que algunas decisiones deben ser tomadas por expertos, las decisiones que versan sobre derecho penal no son parte de este grupo.

En respuesta al argumento de la complejidad, dije que las preguntas centrales del derecho penal no requieren de un gran conocimiento. Todos tenemos creencias más o menos fundadas sobre qué conductas deberían ser reprochadas y sobre cómo deberían ser reprochadas. Más aún, si el derecho penal expresa los valores de una comunidad, nadie está mejor situado para expresarlos que la misma comunidad.

En segundo lugar, afirmé que el argumento de la complejidad asumía que las personas eran incapaces de adquirir conocimiento y perfeccionar sus posiciones durante un proceso deliberativo. Esto surge de la equiparación de la democracia a la agregación de preferencias individuales dadas. Si por el contrario concebimos a la democracia como un proceso en el que los ciudadanos deliberamos intercambiando razones y solamente cuando el tiempo de deliberar haya terminado decidimos, entonces debemos aceptar que nuestro nivel de expertise siempre puede mejorar y alcanzar un nivel tal que nos permita tomar decisiones en materia penal de forma informada.

Por último, en un argumento más simple pero no menos relevante, señalé que los expertos sí participarían si se permitieran 
las iniciativas ciudadanas en materia penal. Ellos seguirían teniendo un lugar central al momento de la toma de decisiones autoritativa dado que las iniciativas de agenda dejan la decisión final en manos del Congreso, por lo que las personas a las que la Constitución actualmente confía la tarea de redactar y sancionar el derecho penal seguirán teniendo la palabra final, pudiendo rechazar los proyectos no satisfactorios. En segundo lugar, la división entre ciudadanía y expertos no es tajante, sino que los expertos son ciudadanos y participan de los movimientos sociales que promueven iniciativas de ley. Por lo tanto, ellos también ocuparán un lugar central a la hora de la redacción de los proyectos que se presentarán de habilitar la iniciativa en materia penal. En consecuencia, sería un error pensar que habilitar la iniciativa ciudadana de ley en materia penal excluiría a los expertos de la formulación del derecho penal.

De esta forma, vemos que existen razones de muy diversa índole que nos permiten rechazar el argumento de la complejidad incluso si aceptamos sus discutibles presupuestos teóricos. Si bien esto no significa que no existan razones que puedan justificar restricciones a la participación ciudadana en materia penal, estas razones no son las brindadas en su origen. Por lo tanto, concluyo que los argumentos de acuerdo a los cuales se sostuvo la restricción acá analizada al momento de la sanción del art. 39 de nuestra Constitución son insuficientes para sostener la restricción a las iniciativas ciudadanas de ley en materia penal.

\section{Bibliografía}

Christiano, T., "Deliberation among experts and citizens" en Parkinson, J. y Mansbridge, J. (eds.), Deliberative systems. Nueva York: Cambridge University Press.

Curato, N., Dryzek, J., Ercan, S., Hendriks, C., y Niemeyer, S. (2017). "Twelve Key Findings in Deliberative Democracy Research" en Dedalus Journal of the American Academy of Arts \& Sciences, Summer 2017, pp. 28-38.

Duff, A. (2015) Sobre el castigo. Por una justicia pena que hable el lenguaje de la comunidad, Buenos Aires: Siglo XXI 
Duff, A. (2015), “¿Hacia una teoría del Derecho penal?”, en Beade, G. y Martí, J. L. (ed.), Discusiones sobre la filosofía del Derecho penal. Bogotá: Universidad Externado de Colombia, pp. 17-68.

Dzur, A (2010). "The Myth of Penal Populism: Democracy, Citizen Participation, and American Hyperincarceration" en The Journal of Speculative Philosophy, Vol. 24, No 4, pp. 354-379.

ELsTER, J. (1997). "The market and the forum" en Bohman, J. y Rehg,

W. Deliberative democracy, Londres: MIT Press.

Fearon, J. (1998) "Deliberation as discussion" en Elster, J. (ed.) Deliberative Democracy, Estados Unidos: Cambridge University Press.

FerRajoli, L (1998). Derecho y razón, Madrid: Trotta

Ferrajoli, L. (2008). Democracia y garantismo, Madrid: Trotta.

Fung, Aaron. "Recipies for Public Spheres: Eight Institutional Design Choices and Their Consequences" en The Journal of Political Philosophy, Vol. 11, Nro. 3, 2003

Gargarella, R. (2016). Castigar al prójimo. Por una refundación democrática del derecho penal, Buenos Aires: Siglo XXI

Goldman, A. y O'Connor, C., "Social Epistemology", The Stanford Encyclopedia of Philosophy (Fall 2019 Edition). Recuperado en https://plato.stanford.edu/entries/epistemology-social/

Gutman, A. y Thompson, D. (2004). Why deliberative democracy. Princeton: Princeton University Press.

Hamilton, A., Madison, J. y Jay, J. (2014) El federalista. México: Fondo de Cultura Económica.

HeIBert, J. L. y Kelly, J. B. (2015). Parliamentary Bill of rights. The eperiences of New Zeland and the United Kingdom, Reino Unido: Cambridge University Press.

Manin, B. (1997) Principios del gobierno representativo, Madrid: Alianza

Pastor, D. (2005) Recodificación penal y principio de reserva de código, Buenos Aires: Ad-Hoc

Peтtт, P. "Is Criminal Justice feasible?", Buffalo Criminal Law Review, Vol. 5, No 2 (January 2002), pp. 427-450

Pratt, J. (2007). Penal populism. Oxon, Canada: Routledge, Taylor and Francis Group 
Pratt, J. y Miao, M. (2017). Populismo penal: el fin de la razón. Nova criminis, Vol 9, $\mathrm{N}^{\mathrm{0}}$ 13, pp. 33-105.

Rawls, J. (1997). "The idea of public reason" en Bohman, J. y Rehg, W. Deliberative democracy, Londres: MIT Press.

SARAT, A y Scheingold, S. A. (2006). Cause lawyers and social movements, California: Stanford University Press.

StrandBrinck, P. (2018). "Epistocracy and participation in a posttruth world". Democratic Theory, vol. 5, no 1, p. 1-17.

SPARKs, R. (2000). "Risk and blame in criminal justice controversies: British press coverage and official discourse on prison security (1993-6)" en Brown, M. y Pratt, J., Dangerous offenders. Estados Unidos: Routledge.

ZafFaroni, E.R. (2014) “¿Habrá llegado la hora?”, Infonews, Buenos Aires, 3 de marzo. 